\title{
Photonic Crystal Fibers
}

\author{
R. BUCZYNSKI* \\ Information Optics Group, Faculty of Physics, Warsaw University \\ Pasteura 7, 02-093 Warsaw, Poland
}

\begin{abstract}
Photonic crystal fibers are a new class of optical fibers. Their artificial crystal-like microstructure results in a number of unusual properties. They can guide light not only through a well-known total internal reflection mechanism but using also photonic bandgap effect. In this paper different properties possible to obtain in photonic crystal fibers are reviewed. Fabrication and modeling methods are also discussed.
\end{abstract}

PACS numbers: 42.81.-i, 42.70.Qs

\section{Introduction}

Photonic crystal fibers (PCFs) are fibers with an internal periodic structure made of capillaries, filled with air, laid to form a hexagonal lattice. Light can propagate along the fiber in defects of its crystal structure. A defect is realized by removing one or more central capillaries. PCFs are a new class of optical fibers. Combining properties of optical fibers and photonic crystals they possess a series of unique properties impossible to achieve in classical fibers.

Classical optical fibers perform very well in telecom and non-telecom applications, but there is a series of fundamental limits related to their structures. The fibers have rigid design rules to fulfill: limited core diameter in the single-mode regime, modal cut-off wavelength, limited material choice (thermal properties of core glass and cladding glass must be the same).

The design of PCFs is very flexible. There are several parameters to manipulate: lattice pitch, air hole shape and diameter, refractive index of the glass, and type of lattice. Freedom of design allows one to obtain endlessly single mode fibers, which are single mode in all optical range and a cut-off wavelength does not exist. Moreover there are two guiding mechanisms in PCF: index guiding mechanism (similar to the one in classical optical fibers) and the photonic bandgap mechanism.

*e-mail: rbuczyns@igf.fuw.edu.pl 
By manipulating the structure it is possible to design desired dispersion properties of the fiber. PCFs having zero, low, or anomalous dispersion at visible wavelengths can be designed and fabricated. The dispersion can also be flattened over a very large range. Combining anomalous dispersion with small mode field areas results in outstanding nonlinear fibers. On the other hand large, solid or air core single mode fibers can be achieved.

The idea of a photonic crystal fiber was presented for the first time by Yeh et al. [1] in 1978. They proposed to clad a fiber core with Bragg grating, which is similar to $1 \mathrm{D}$ photonic crystal. A photonic crystal fiber made of $2 \mathrm{D}$ photonic crystal with an air core was invented by P. Russell in 1992 and the first PCF was reported at the Optical Fiber Conference (OFC) in 1996 [2]. A short overview of PCF development is presented in the Table.

TABLE

Overview of photonic crystal fibers development.

\begin{tabular}{l|l}
\hline \hline 1978 & Idea of the Bragg fiber \\
1992 & Idea of the photonic crystal fiber with air core \\
1996 & Fabrication of a single-mode fiber with photonic coating \\
1997 & Endlessly singe mode PCF \\
1999 & PCF with photonic bandgap and air core \\
2000 & Highly birefringent PCF \\
2000 & Supercontinuum generation with PCF \\
2001 & Fabrication of a Bragg fiber \\
2001 & PCF laser with double cladding \\
2002 & PCF with ultra-flattened dispersion \\
2003 & Bragg fiber with silica and air core
\end{tabular}

\section{Guidance mechanisms in photonic crystal fibers}

If the defect of the structure is realized by removing the central capillary, then guiding of an electromagnetic wave in a photonic crystal fiber can be regarded as a modified total internal reflection mechanism. The modification is due to the network of air capillaries that leak higher modes so that only one fundamental mode is carried. This is the mode with the smallest diameter, close to the size of the defect, i.e., to the lattice constant of the periodic structure [3, 4].

According to Ref. [2] a fiber is single-mode if $d / \Lambda<0.4$, where $d$ is the diameter of the air channel and $\Lambda$ is the crystal lattice constant. The guiding of light in a photonic crystal fiber was first demonstrated in 1996 in a solid-core fiber (solid core guidance). 
In a lattice of air capillaries, the central one was replaced by a rod $[2,3]$. If the central defect is realized by inserting a central air capillary, which has a diameter different than other capillaries (usually bigger), then we can obtain a photonic bandgap (PBG). Light guidance is then an analogue of a mechanism known in solid state physics as the electron conduction mechanism in materials with an energy-band structure. In 1997 the guiding of light in an air defect was demonstrated (hollow core PGB guidance). A few central capillaries were removed from a hexagonal lattice leaving a large hole filled with air [5].

Periodically distributed air cores can form an artificial 2D photonic crystal structure with lattice constant similar to the wavelength of light. In the $2 \mathrm{D}$ crystal structures photonic bandgaps exist that prevent propagation of light with a certain range of frequencies. If periodicity of the structure is broken with a defect (lack of air cores or large air core) a special region with optical properties different from the photonic crystal is created. The defect region can support modes with frequencies falling inside photonic bandgap, which prevent them from penetration of photonic crystal. The modes are strongly confined to the defects and guided along them through the fiber. Since photonic bandgap is responsible for confinement of the light in the core, it is not required that the defect region has a higher refractive index than the surrounding.

\section{Fabrication methods}

Whatever the type of glass and the type of structure, the principal method of fabrication of photonic crystal fibers is multiple thinning. However, there are also reports on fabrication of PCF with extrusion processes, which is mainly used for soft glass PCF formation. In this method, molten glass is forced through a die containing a designed pattern of holes [6].

The multiple thinning method is well known in the technology of image-guiding structures performance (fiber plates, image guides, etc.) [7] (Fig. 1). With this method, PCFs are fabricated in a several step process (Fig. 2).

In the first step individual capillaries are created. One can use capillaries with different diameters and wall thickness (this influences the $d / \Lambda$ ratio in the fiber), different cross-sections (circular, hexagonal, square) and different types of glass (silica, silicate, multi-component with various composition of oxides, etc.) (Fig. 3). Next, individual capillaries are positioned, by hand, to make a multicapillary preform with the required symmetry. The defect in which light propagates is a glass rod or, in the case of fibers with a photonic bandgap, a hole with a suitable diameter. These defect rods are positioned in the structure. A structure so prepared is then fused and drawn with a fiber drawing tower to a millimeterscale and usually named an intermediate preform. This is a thermally integrated glass rod with holes at the locations of the capillaries and filled spaces in between. In order to obtain a fiber of a given diameter and required structural parameters 


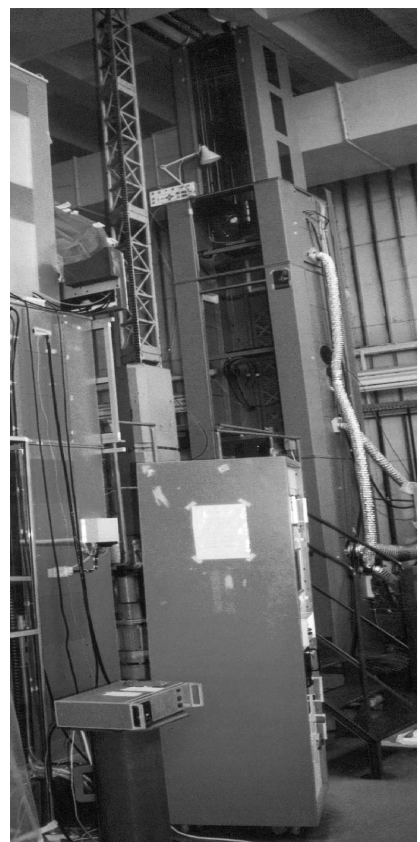

Fig. 1. The fiber drawing tower for PCF fabrication at the Institute of Electronic Materials Technology, Warsaw, Poland.

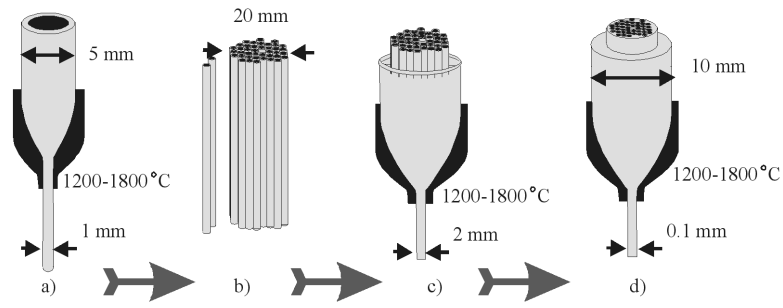

Fig. 2. Photonic crystal fiber fabrication: (a) creation of individual capillaries, (b) formation of the preform, (c) drawing of intermediate preform, (d) drawing of the final fiber.

(distance between holes, hole diameter, core diameter) the intermediate preform is complemented by extra glass rods. Such an intermediate preform is then fused and drawn again with a fiber drawing tower to a final fiber with micrometer-scale structure. Finally, extra layers of polymer are usually added in this process to create a coating protecting the fiber mechanically.

During the experiments it is observed that the thinning of a structure with a given symmetry affects the shape of the holes' cross-section. When the wall thickness is small, they tend to adopt a shape corresponding to the symmetry of the lattice. For a hexagonal lattice, it is a hexagon while for a square lattice it is a square. This is apparent when the thinning is applied to highly viscous glass (low 


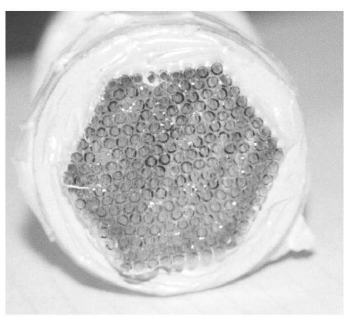

a)

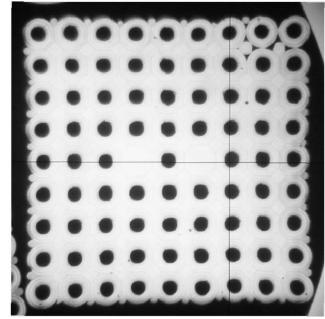

d)

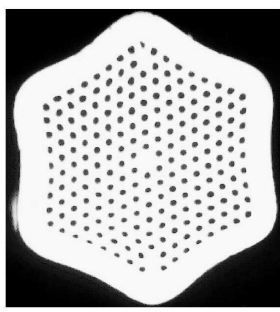

b)

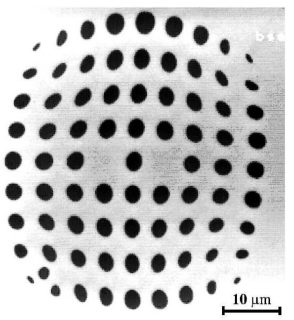

e)

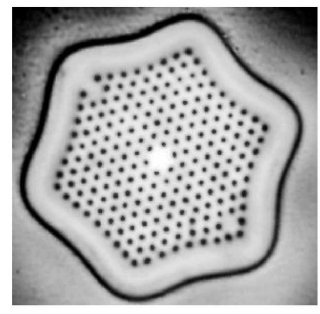

c)

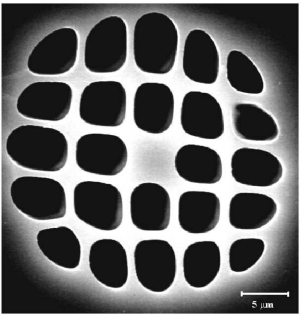

f)

Fig. 3. Fabrication of photonic crystal fibers: preforms, intermediate preforms and final fibers (Institute of Electronic Materials Technology (IEMT)): (a) the preform of PCF with holes $\oslash=1 \mathrm{~mm}$ with hexagonal lattice; (b) the intermediate preform with air holes $\oslash=250 \mu \mathrm{m}$ with hexagonal lattice; (c) the PCF fiber; diameter of the fiber $\oslash=120 \mu \mathrm{m}$, air holes diameter $d=3 \mu \mathrm{m}, d / \Lambda=0.5$; (d) the intermediate preform of double core PCF with square lattice; (e) a double core PCF fiber with a square lattice, diameter of the fiber $250 \mu \mathrm{m}$, air holes diameter $d=2.5 \mu \mathrm{m}, d / \Lambda=0.5$; (f) a multimode PCF fiber with a square lattice; diameter of the fiber $160 \mu \mathrm{m}$, air holes diameter $3 \mu \mathrm{m}$.

temperature) and to structures with a high $d / \Lambda(>0.6)$ ratio (Fig. 3f). The same phenomenon is evident in the thinning of multi-fiber structures (image guides).

Obtaining photonic fibers with required transmission characteristics is a difficult technological problem. One has to shape structures of microscopic size controlling only macroscopic parameters such as temperature and stretching rate. Defects are encountered affecting properties of the structure that deviate from their ideal values assumed in the simulations.

The main fabrication problems are presence of deformed air holes, emergence of additional holes, and perturbations of the structure's symmetry (Fig. 4) [8]. The presence of holes with different diameters and irregular shapes is clearly visible in structures with a square lattice. Usually the temperature in the fiber is nonuniform and has a radial distribution. As a result, the outer holes become more deformed and have smaller diameters. It is therefore recommended to add two or three rings of capillaries around the originally designed structure. Those additional capillaries do not affect the mode guided in the defect. Emergence of additional holes is often present when the spaces between capillaries do not close during the 


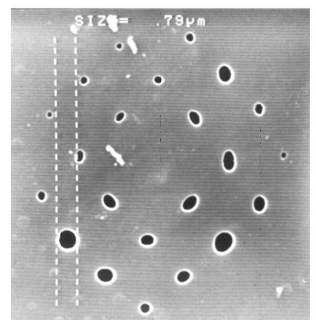

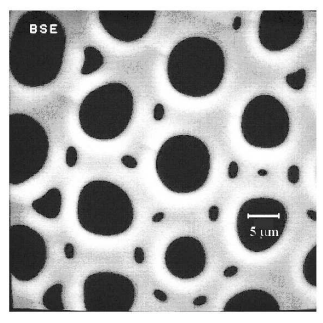

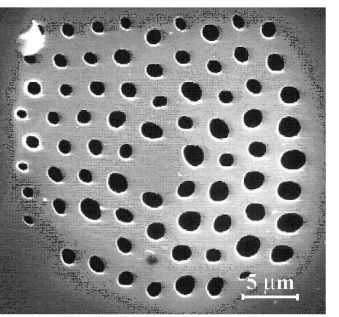

Fig. 4. Defects in PCF fabrication: (a) the $5 \times 5$ square structure with different hole diameters; (b) the hexagonal structure with gaps between capillaries not properly closed; (c) the $9 \times 9$ square structure with displaced holes.

thinning process. Perturbation of the structure's symmetry is observed, especially in a square lattice, where holes may be displaced (tending to adopt triangular symmetry), the structure may be skewed or the rows of holes may be undulated.

Avoiding such flaws requires a precise control of all thinning processes (capillaries, intermediate preform). It is essential to properly adjust and control the temperature of the thinning process, the temperature distribution in the oven, centering of the preform guidance, the speed of feeding and pulling. These parameters determine the diameter of the fiber, the temperature distribution over its cross-section and the heating time.

Most reports on PCFs have described fibers made of silica glass. Silica provides very good fiber performance for most applications with wavelengths in the range of 200-2500 nm, but using other materials can enhance specific parameters of the fibers and transmission outside this spectral region. Therefore, more and more attention is also paid to fibers drawn from multicomponent glass: tellurite, fluoride, and chalcogenide ones. Multicomponent glass offers several useful properties not possessed by silica, such as a high refractive index, good infrared transmittance, high optical nonlinearity and relatively low photon energy. Several fibers made of silicate [8], chalcogenite [9], and tellurite glass [10] have been reported. The silicate glass can be doped much more and therefore the range of possible modifications of their optical and mechanical characteristics is much wider. The high attenuation in glass of this type is usually considered to be a great disadvantage. However, this property is unimportant wherever short lengths of fiber are used, e.g. in sensors.

\section{Modeling methods}

Commonly used methods for modeling of optical fibers cannot be applied successfully in PCF modeling. These fibers have a high refractive index contrast and a subwavelength periodical structure. Therefore, the methods used in modeling photonic crystals and electromagnetic fields are adapted to this purpose. 
The finite difference time domain (FDTD) method is widely used for calculation of the evaluation of an electromagnetic field in depressive media [11]. The wave propagation through the PCF structure is found by direct integration in the time domain of Maxwell's equations in a discrete form. Space and time is discrete in a regular grid. Evaluation of the electrical and magnetic field is calculated on a Yee cell (Fig. 5). In addition the boundary conditions are added (absorbing or periodic ones). Most often uniaxial perfect matching layer (UPML) boundary conditions are used for PCF modeling. The method allows obtaining transmission and reflection coefficients, energy flow of propagation fields (Poynting vector). It allows the observation of a steady state field distribution as well as the temporary field distribution.

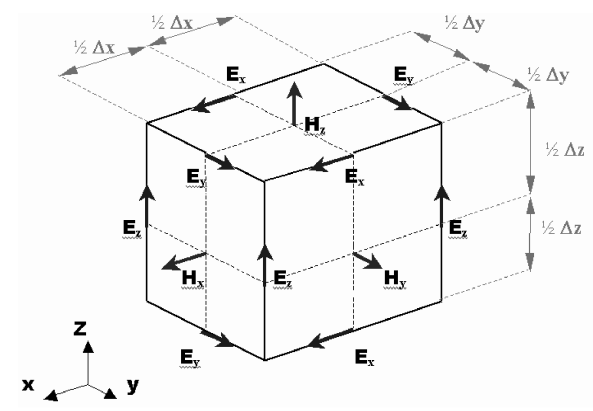

Fig. 5. The Yee cell describes all components of electrical and magnetic field in a cube. Every component of the electromagnetic field is defined only in one place in the unit Yee cell.

The FDTD method is universal, robust, and methodologically simple. The main drawback of this method is very high time and memory complexity of the algorithm. Since PCF are 3D structures with 2D refractive index distribution only short pieces of the fiber can be simulated with these methods. It can be successfully applied to model tapers, couplers, and double core coupling in the PCFs. Large volume simulations can be performed with computer clusters because the FDTD method can be relatively easily implemented as a parallel algorithm.

Similar discretization schemes can be used in the context of the beam propagation method (BPM) [12] or finite difference (FD) mode solvers. Zhu et al. use the 2D Yee discretization scheme in their full-vector finite difference mode solver [13] to express transversal electrical and magnetic field discrete form. By applying the finite difference procedure, the full vectorial wave equation yields the algebraic eigenproblem.

The full-vector plane wave expansion (PWE) method offers a very efficient and straightforward approach to model PCFs [14, 15]. This method allows solving the full-vector wave equation for the magnetic field. In this model a periodic field as well as a position-dependent dielectric constant are represented using Fourier 
expansion in terms of harmonic functions defined by the reciprocal lattice vector. From Maxwell's equations the full-vector wave equation is obtained for magnetic field $\boldsymbol{H}_{\boldsymbol{k}}$ :

$$
\nabla \times\left[\frac{1}{\varepsilon(\boldsymbol{r})} \nabla \times H_{\boldsymbol{k}}\right]=-\frac{\omega^{2}}{c^{2}} \boldsymbol{H}_{\boldsymbol{k}}
$$

where $\boldsymbol{k}$ is the wavevector, and $\varepsilon(\boldsymbol{r})$ is a value of the dielectric constant in the structure. A simulated PCF structure is represented as a periodic supercell, which contains a crystal structure and its defects. Due to the periodicity we can express $\boldsymbol{H}_{\boldsymbol{k}}$ as a sum of plane-waves based on Bloch theorem:

$$
\boldsymbol{H}_{\boldsymbol{k}}=\sum_{\boldsymbol{G}} h_{\boldsymbol{k}-\boldsymbol{G}} \exp (-\mathrm{i}(\boldsymbol{k}-\boldsymbol{G}) \cdot \boldsymbol{r}),
$$

where $\boldsymbol{G}$ is a lattice vector in reciprocal space. The dielectric constant $\varepsilon(\boldsymbol{r})$ is represented with a Fourier expansion: $\frac{1}{\varepsilon(\boldsymbol{r})}=\sum_{\boldsymbol{G}} V_{\boldsymbol{G}} \exp (\mathrm{i} \boldsymbol{G} \cdot \boldsymbol{r})$, where $V_{\boldsymbol{G}}=$ $\frac{1}{A_{\mathrm{u}}} \int \frac{1}{\varepsilon(\boldsymbol{r})} \exp (-\mathrm{i} \boldsymbol{G} \cdot \boldsymbol{r}) \mathrm{d} \boldsymbol{r}$ and $A_{\mathrm{u}}$ is the area of a unit cell.

Substituting (2) and (3) into wave equation (1) algebraic eigenproblem is obtained. Solving the eigenvalue problem all frequencies of allowed modes can be found.

The PWE allows calculating dispersion relations and photonic bandgap in periodic dielectric structures (Fig. 6). It can be applied to any type of crystal structure, including irregular crystals. It allows finding photonic band structure in the PBG guidance mechanism, as well as modes in the effective index guidance mechanism. It is an accurate, relatively fast method, however it has several drawbacks. It cannot be used for calculating structures with active materials (absorption and amplification). Also, it does not bring any information about scattering losses, transmission and reflection of incident light in the PCF.

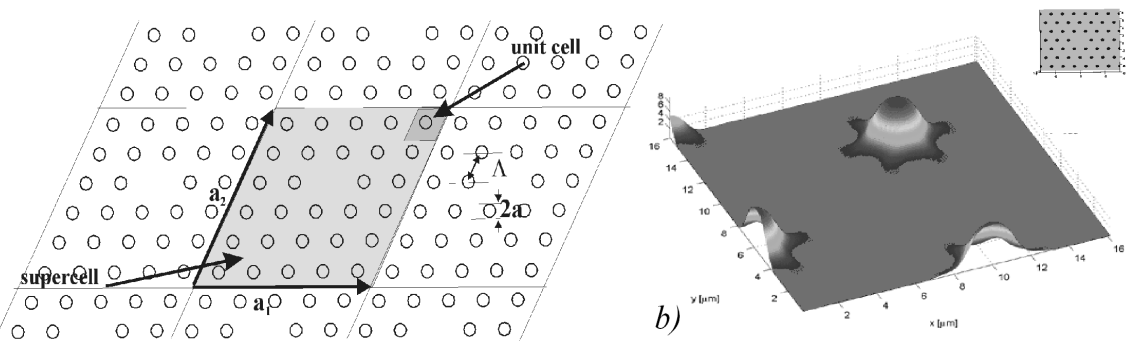

Fig. 6. PWE simulation: (a) the PCF structure is represented as a periodic supercell, which contains crystal structure and its defects; (b) an example of simulation results with PWE - intensity distribution in periodic supercells.

The method of localized basis functions (LBF) is based on direct solution of Maxwell's equations, similarly to the PWE method [16]. It assumes that guided modes of PCF are localized in the close area around the crystal defect and the 
modes can be described as a sum of Hermite-Gaussian functions localized in the neighborhood of the core. It allows reducing dramatically the number of base functions and computational complexity. In the LBF method a medium translationally invariant along $z$ the axis is assumed and Maxwell's equations are written as wave equations for transversal magnetic field:

$$
\left(\nabla_{\perp}^{2}+k^{2} \varepsilon\right) h_{\perp}+\left(\nabla_{\perp} \ln (\varepsilon)\right) \times\left(\nabla_{\perp}+h_{\perp}\right)=\beta^{2} h_{\perp},
$$

where $\nabla_{\perp}$ is the gradient in the transversal plane and $h_{\perp}$ are transversal components of the magnetic field $H_{i}, i=x, y$ :

$$
H_{i}=h_{i} \exp (\mathrm{i}(\beta z-c k t)),
$$

base functions are built of the set of Hermite-Gaussian functions:

$$
\phi_{m n}=\exp \left(-\frac{x^{2}+y^{2}}{2 \Lambda^{2}}\right) H_{m}\left(\frac{x}{\Lambda}\right) H_{n}\left(\frac{y}{\Lambda}\right),
$$

where $H_{m}$ is the Hermite polynomial of the order $m$. The functions $\phi_{m n}$ are orthogonal and create a complete system of the base. The wave equation (3) can be noted in the following algebraic form:

$$
\sum_{k l} L_{k, l}^{m, n} h_{\perp}^{k, l}=\beta^{2} h_{\perp}^{m, n}
$$

where $L_{k, l}^{m, n}$ are the matrix coefficients of the left-hand side operator in (3) and $h_{\perp}^{k, l}$ is the transversal magnetic field in Hermite-Gaussian basis. Solving eigenvalue problem propagation constant $\beta$ and field distribution can be obtained. This method has been developed for modeling PCF with circular holes and hexagonal lattice. It allows modeling of modal and dispersion properties of the PCFs.

The supercell lattice method is a combination of PWE and LBF methods [17]. The electric field is decomposed by use of Hermite-Gaussian functions. PCF is decomposed on two virtual different periodic dielectric structures of perfect photonic crystals. The first one represents a photonic crystal of photonic cladding, while the second photonic crystal represents central defects of the PCFs. The structures of both virtual PCs are expanded in cosine functions. From the wave equation and the orthonormality of the Hermite-Gaussian functions, the propagation characteristics of the PCFs, such as the mode field distribution, the effective area, and the dispersion property, are obtained.

The full-vector finite element method (FEM) has been applied to PCF modeling successfully [18]. It allows calculating both dispersion and confinement properties of PBG and solid core structures. For a given frequency the method provides us with a complex propagation constant $\gamma(\omega)=\beta(\omega)+i \alpha(\omega)$, where $\beta$ is the standard propagation constant of the plane-wave along the fiber and $\alpha$ is the attenuation constant associated with the exponential decay along the fiber axis.

A multipole method originally developed for modeling diffractive structures and photonic crystals has been successfully used for index and photonic bandgap 
guiding PCFs [19]. In this method the field is written in terms of cylindrical harmonics centered around each air hole. The method has advantages in terms of speed and accuracy. Since finite cladding is assumed, the calculations can be performed in this way.

Apart from the above-mentioned methods there are several other ones used for PCF modeling: scattered matrix method, transferred matrix method and others [20]. However mostly used are PWE, FD, and multipole methods for modeling properties of PCFs.

\section{Properties of single mode photonic crystal fibers}

\subsection{Endlessly single mode fibers}

PCF can be designed so that they are single mode for a large range of visual and near infrared spectrum. Classical step index fibers (SIFs) always have a cut-off frequency above which the fibers starts to be multimode.

To determine the number of guided modes in SIF usually a normalized frequency $V$ is used. $V$ is defined as:

$$
V=\frac{2 \pi \rho}{\lambda} \sqrt{n_{\text {core }}^{2}-n_{\text {cladding }}^{2}},
$$

were $\rho$ is the core radius, $n_{\text {core }}$ and $n_{\text {cladding }}$ are refractive indexes of the core and the cladding, respectively.

In the case of standard fibers, the cladding index is almost wavelength independent and $V$ increases when wavelength decreases. It results in multimode operation regime for cut-off normalized frequency higher than 2.405 .

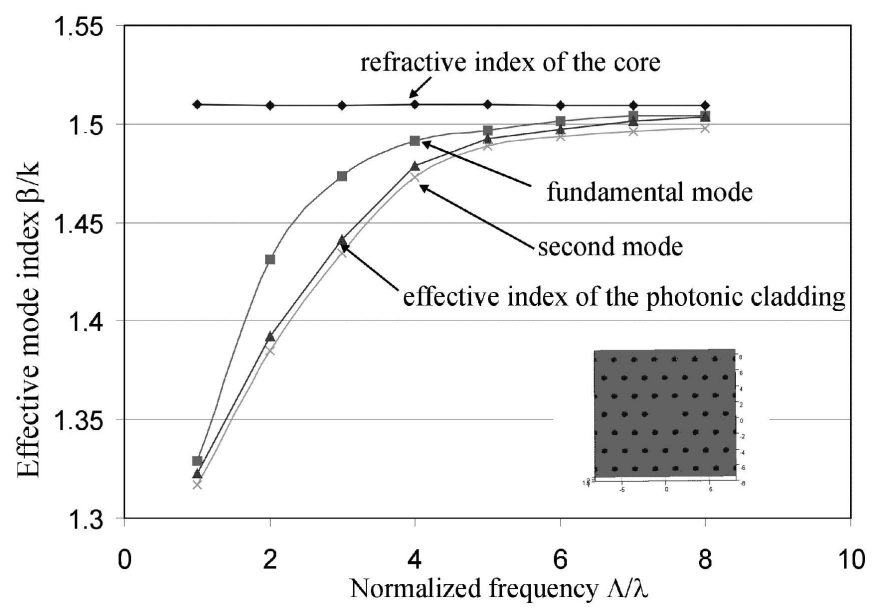

Fig. 7. Modal properties of endlessly single mode PCF made of multicomponent glass. For any wavelength a mode index value of the modes, higher than the fundamental one, is lower than the effective cladding index. 


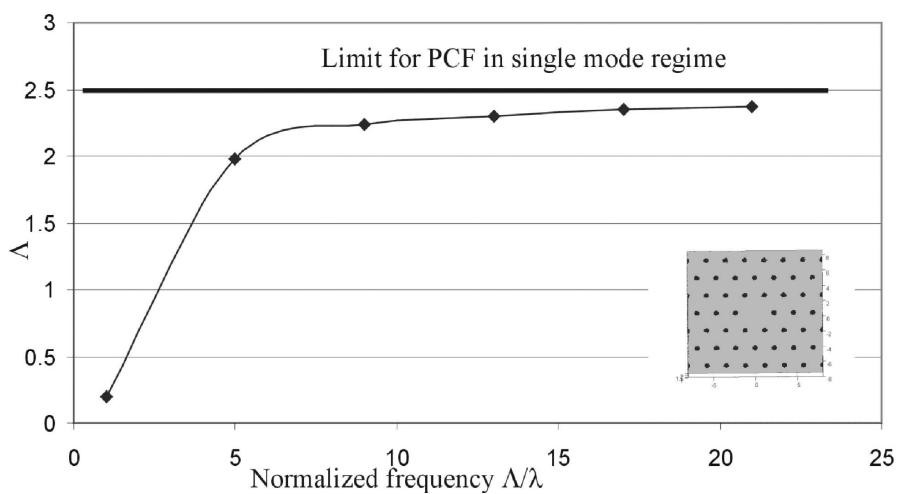

Fig. 8. Normalized frequency for hexagonal lattice PCF, filling factor 0.20. The PCF guides only the fundamental mode in the visible and near infrared regions.

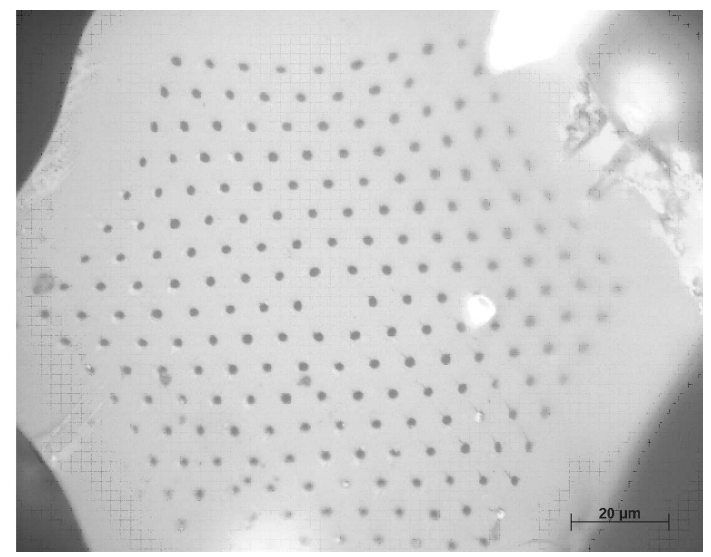

Fig. 9. Typical structure of a single mode PCF. Fiber fabricated at IEMT. Endlessly single mode PCF has a filling factor less that 0.2 .

For PCF a value of the effective refractive index of photonic cladding depends strongly on wavelength, while in classical fibers it was almost constant (Fig. 7). The normalized frequency tends to a stationary value for short wavelengths. A refractive index of photonic cladding and therefore stationary value of normalized frequency is defined by the cladding structure, namely by the fill factor (the ratio of the hole diameter $d$ to the period of the lattice $\Lambda$ ). With a proper design it is possible to keep $V$ below a cut-off normalized frequency for any wavelength range (Fig. 8).

The PCF, that fulfills this condition, is usually named as endlessly single mode. A cut-off normalized frequency for PCF has been estimated as 2.5 [21, 22]. A typical structure of endlessly single mode fiber is presented in Fig. 9. 


\subsection{Large mode area}

Conventional fibers have a strong limit on the core size and the numerical aperture (NA) in a single mode regime. For any wavelength and core diameter there is a maximum NA which makes a single mode regime of operation possible. The value of NA is controlled by the difference in refractive index of the core and the cladding. The fabrication of a standard SIF with a large mode area would require refractive index control in chemical vapor deposition CVD with a very high accuracy $\left(10^{-6}\right.$ and more), which limits the mode field diameter (MFD) in practice. Usually the MFD of conventional SIF, defined as $1 / e$ width in intensity, is about $9 \mu \mathrm{m}$ for $1.55 \mu \mathrm{m}$ wavelength.

In case of PCF MFD can vary in a single mode regime, depending on requirements. Large mode areas can be engineered by increasing the lattice pitch of the photonic cladding, decreasing the air hole diameter or increasing the size of the defect in photonic cladding (removal of more than one of the central air holes).

As it was shown by Bagget et al. [23] large core SIF and PCF can have a similar MFD at any particular wavelength in case of PCF with a single capillary defect. However, PCF remains single mode over a large range of frequencies, while SIF starts to be multimode close to the designed wavelength.

A typically large mode PCF has a MFD of 9-26 $\mu \mathrm{m}$ and is single mode for all wavelengths. The MFD $9 \mu \mathrm{m}$ can be obtained for SIFs at 1.55, but for wavelength of $400 \mathrm{~nm}$ the same PCF offers ten times larger core than it is possible in standard fibers. PCFs with large MFD are obtained in the structures where the core consists of several rods that replaced air-holes. In practice, the band losses limit the use of large core single mode fibers. However, it does not limit PCF performance since Bagget et al. [23] have shown that conventional fibers and PCF with similar mode areas experience similar band losses.

\subsection{Dispersion properties}

In SIFs the total dispersion comes from material and waveguide dispersion. Unlike conventional fibers the photonic crystal waveguide dispersion can be very strong. Furthermore, the "material dispersion" is modified by artificial photonic cladding with the presence of air-holes. Photonic crystal cladding changes massively over a narrow range of wavelength. A key parameter that describes properties of fibers is a group velocity dispersion (GVD). It is defined as:

$$
G V D=\frac{\lambda}{c} \frac{d^{2} n_{\mathrm{eff}}}{d \lambda^{2}}
$$

where $n_{\text {eff }}$ is the effective refraction index $n_{\mathrm{eff}}=\frac{\beta\left(\lambda, n_{m}(\lambda)\right)}{k_{0}}$.

Dispersion characteristics in PCFs can be easily shaped due to the flexibility of varying air-hole size and the position in the photonic cladding. It is not possible to obtain analogue properties with SIF, since there are rigid limits on parameter modification when the single mode operation regime is required. Varying lattice 


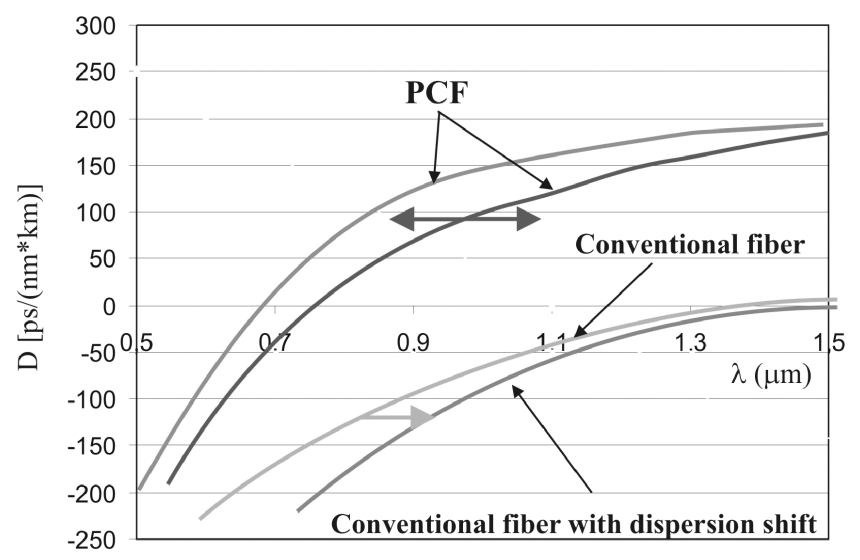

Fig. 10. A comparison of dispersion in SIF and in an index-guiding PCF.

pitch and air-hole sizes in PCFs a zero-dispersion wavelength can be shifted into the visible region [24] (Fig. 10). In case of conventional fibers the zero-dispersion wavelength is limited at a short wavelength side to about $1.3 \mu \mathrm{m}$ and can be shifted only into longer wavelengths. The fibers with a shifted dispersion are obtained with lower refractive index ring around the core - W-type structure.

If the zero-dispersion wavelength is in the visible region, it automatically gives a positive (anomalous) dispersion in the visible range. PCF with a positive dispersion can be used for dispersion compensation in the telecommunication lines.

Also PCF with a flat dispersion can be obtained [24]. The PCF with hexagonal lattice and lattice pitch $\Lambda=2.62 \mu \mathrm{m}$ and air-holes of the diameter $a=0.316 \mu \mathrm{m}$ has a flatness of $2 \mathrm{ps} /\left(\mathrm{km}^{*} \mathrm{~nm}\right)$ is reported in the range of

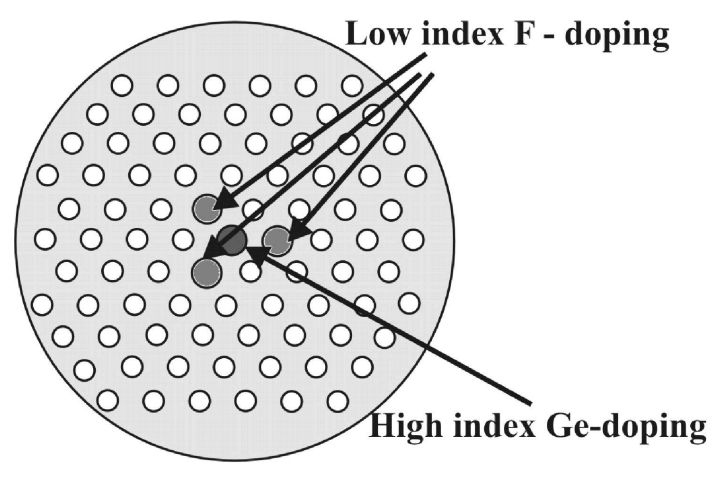

Fig. 11. A new concept of PCF after Hansen [26]. The central part of the core has a higher refractive index and three outer rods in the core have lower refractive indices. The core is surrounded with hexagonal photonic cladding. By varying parameters of the structure different dispersion characteristics are achievable. 
1.3-1.9 $\mu \mathrm{m}$. Disadvantage of the design proposed by Ferrando et al. [26] is a high attenuation [25]. In case of conventional SIFs with a modified flat dispersion a maximum $300 \mathrm{~nm}$ bandwidth can be obtained.

To overcome this drawback a new design of $\mathrm{PCF}$ with triangular core is proposed by Hansen [26]. A central part of the core has a slightly higher refractive index due to Ge-doping and is surrounded by three $F$-doped regions with a lower refractive index (Fig. 11). The core is surrounded with hexagonal photonic cladding. By varying parameters of the structure, different dispersion characteristics are achievable. An alternative design is proposed by Saitoh et al. [27]. A flatness of $0 \pm 0.5 \mathrm{ps} /\left(\mathrm{km}^{*} \mathrm{~nm}\right)$ is predicted in the range of $1.19-1.69 \mathrm{~mm}$ with relatively low attenuation.

\section{Hollow core fibers}

Hollow core fibers consist of a hollow core surrounded by micro structured cladding with a periodic arrangement of air-holes in glass. There are two types of hollow core fibers: the first one has a photonic cladding made of periodically distributed air-holes [28] (2D photonic crystal), the second one has a photonic cladding made of circularly arranged air cells [29] (Bragg reflectors, 1D photonic crystal) (Fig. 12). In both cases total internal reflection conditions are not fulfilled since the air core has a lower refractive index than the cladding. Light can propagate along the fiber only with photonic bandgap mechanism.
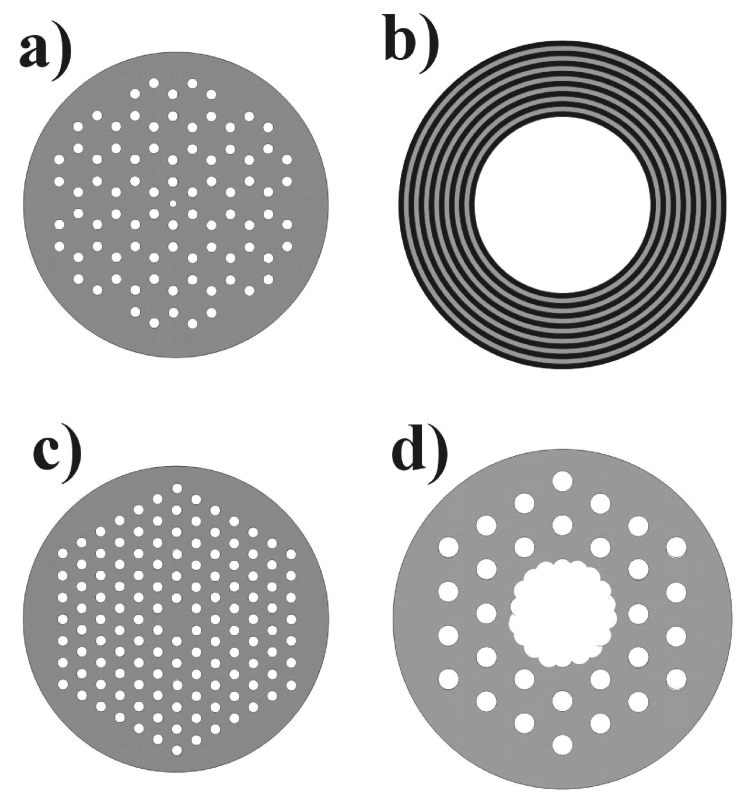

Fig. 12. Different types of photonic bandgap PCFs: (a) honeycomb photonic bandgap fiber (after Broeng et al. [28]), (b) Bragg hollow core fiber (after Vienne et al. [29], (c) hexagonal lattice solid-core photonic bandgap fiber, (d) large hollow core hexagonal lattice fiber (after Mangan et al. [30]). 
Since only a small fraction of light propagates in glass, all effects related to interaction between glass and light (dispersion, scattering, nonlinear effects) are drastically reduced. It is expected to achieve an ultra-low transmission.

A recently reported hollow fiber with $2 \mathrm{D}$ photonic cladding has attenuation equal to $1.7 \mathrm{~dB} / \mathrm{km}$ at $1.56 \mu \mathrm{m}$ wavelength over $800 \mathrm{~m}$ test samples [30]. It was built on a hexagonal lattice with a $20 \mu \mathrm{m}$ diameter hollow core created by removal of nineteen central capillaries (Fig. 12d). It is a large improvement in respect to the PCF reported previously where attenuation was equal to $13 \mathrm{~dB} / \mathrm{km}$ [31], however still one order of magnitude more than conventional state-of-the-art silica fibers with attenuation of $0.15 \mathrm{~dB} / \mathrm{km}$ [32].

A large core allows $99 \%$ of energy to be transmitted into the air. Because of this, most physical reasons of attenuation existence in the fiber are diminished. In conventional silica fibers attenuation is caused by Rayleigh scattering at short and multi-photon absorption at long wavelengths in the bulk material. It gives a minimum attenuation at around $1550 \mathrm{~nm}$. In hollow core, since most of the energy is transmitted through air, the effects of Rayleigh scattering and multi-photon absorption are drastically reduced. Internal surfaces between core and photonic cladding, their roughness and size variation become the main source of attenuation in hollow fibers.

The recently reported first hollow-core air-silica Bragg fiber [29] transmits over the wavelength range of $0.4-2.2 \mathrm{~mm}$ and has attenuation of $10 \mathrm{~dB} / \mathrm{km}$. The wavelength of maximum transmission was varied from around $1.4 \mu \mathrm{m}$ for a $120 \mu \mathrm{m}$ fiber to $0.95 \mu \mathrm{m}$ for a $80 \mu \mathrm{m}$ fiber.

It is very likely that hollow-core fibers can transmit with attenuation of $0.95 \mathrm{~dB} / \mathrm{km}$ at $1950 \mathrm{~nm}$. Attenuation of PCF should decrease with $\lambda^{3}$ and it will be limited by absorption of infrared in glass, where the fraction of energy propagates $(0.25-1 \%)$.

Apart from their telecom applications low transmission losses hollow-core fibers are very promising in other areas. Since nonlinear effects in the core are negligible these fibers are extremely interesting candidates as media for large-energy delivery, both continuous and pulse. Transmission of $2 \mathrm{MW}$ peak power optical solitons over a distance of $200 \mathrm{~m}$ was reported in [33]. The power is two orders of magnitude higher than that transmitted in conventional fibers.

Also exotic wavelengths which experience high attenuation in the glass can be successfully transmitted through this kind of the fiber. It is of great interest in medicine, biology, and spectrometry, where broad band spectrum of light or specific wavelengths have to be transmitted. Hollow core can be also filled with liquid crystals or a variety of gases [34]. Several applications of such structures in sensing are proposed. Particle levitation in hollow-core fiber over $150 \mathrm{~mm}$ distance with $80 \mathrm{~mW}$ laser power has been also reported [35]. It shows a possibility of transportation of micro-particles over long distances in a controlled way. 


\section{Fibers with special properties}

\subsection{Double-core fibers}

Structures with two or more cores made with traditional step-index technology are useful in many applications like, for example, directional couplers, wavelength multiplexers/demultiplexers, and band sensors. With the multicapillary fabrication technique it is easier to form multi-core PCF structures than the traditional step-index ones. A design of required coupling and dispersion characteristics of multi-core PCFs is possible by selecting a suitable crystal lattice of the fiber, shapes of holes, and positions of the cores, i.e., defects in the lattice structure. In a single fiber one can have cores with dissimilar diameters and properties.

Transmission properties of a double-core PCF were investigated by Mangan et al. [36]. It can be used as directional coupler with mechanical and thermal switching [37] as well as band [38] and strain sensors [39]. Highly birefringent double-core PCFs are also used as polarization splitters [40].

In double-core PCF we assume an existence of two crystal defects in lattice nodes in the form of solid glass instead of air holes (Fig. 13). Two solid cores are separated by a single air hole. A fundamental mode in double-core structure consists of four components, with two components for orthogonal polarizations. For every polarization there are even and odd mode components.

For both orthogonal polarizations the coupling length $L$ is obtained individually from propagation constants of even and odd components of the fundamental mode as:

$$
L_{x, y}=\frac{\pi}{\beta_{\text {even }, x, y}-\beta_{\text {odd }, x, y}}=\frac{\lambda}{2\left(n_{\text {even }, x, y}-n_{\text {odd }, x, y}\right)},
$$

where $\beta_{\text {even }, x, y}$ and $\beta_{\text {odd }, x, y}$ are propagation constants for orthogonal polarization components in the fundamental mode; $n_{\text {even }, x, y}$ and $n_{\text {odd }, x, y}$ are mode indexes of
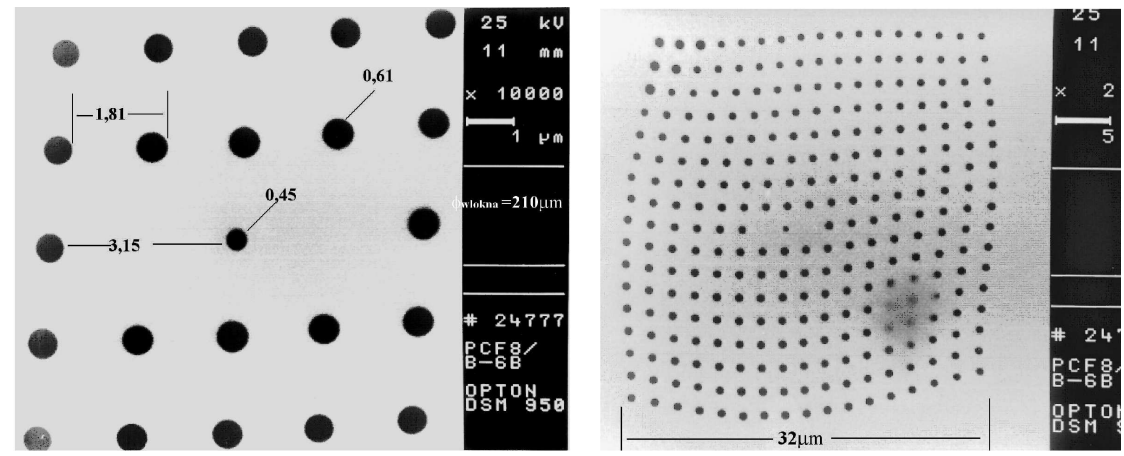

Fig. 13. The cross-sections of the double-core PCF fiber with a square lattice, fiber's diameter of $220 \mu \mathrm{m}$, the lattices constant of $\Lambda=1.81$, the hole diameter $d=0.61 \mu \mathrm{m}$ $(d / \Lambda=0.34)$ and the central hole diameter $d_{\mathrm{c}}=0.45 \mu \mathrm{m}$. The samples fabricated at IEMT. 
even and odd components for orthogonal polarizations in the fundamental mode. In general, four components of the fundamental mode can have different propagation constants. As a result the PCF is birefringent and has different coupling lengths, $L_{x}$ and $L_{y}$, for both orthogonal polarization components (Fig. 14). However, for every structure the size of the central air hole can be adjusted to obtain

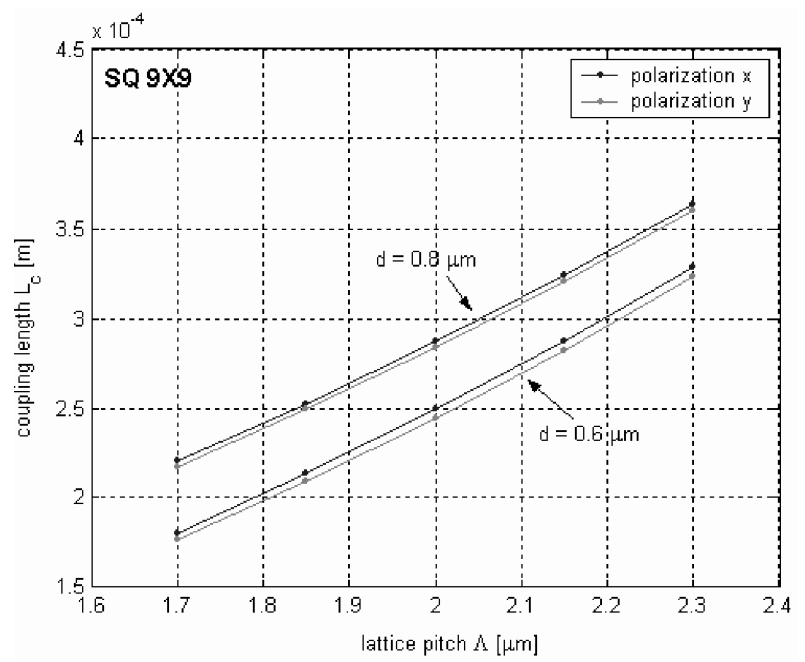

Fig. 14. The coupling length in a function of lattice constant for hexagonal lattice and for different diameter of the air holes $d=0.6 \mu \mathrm{m}, d=0.8 \mu \mathrm{m}$.
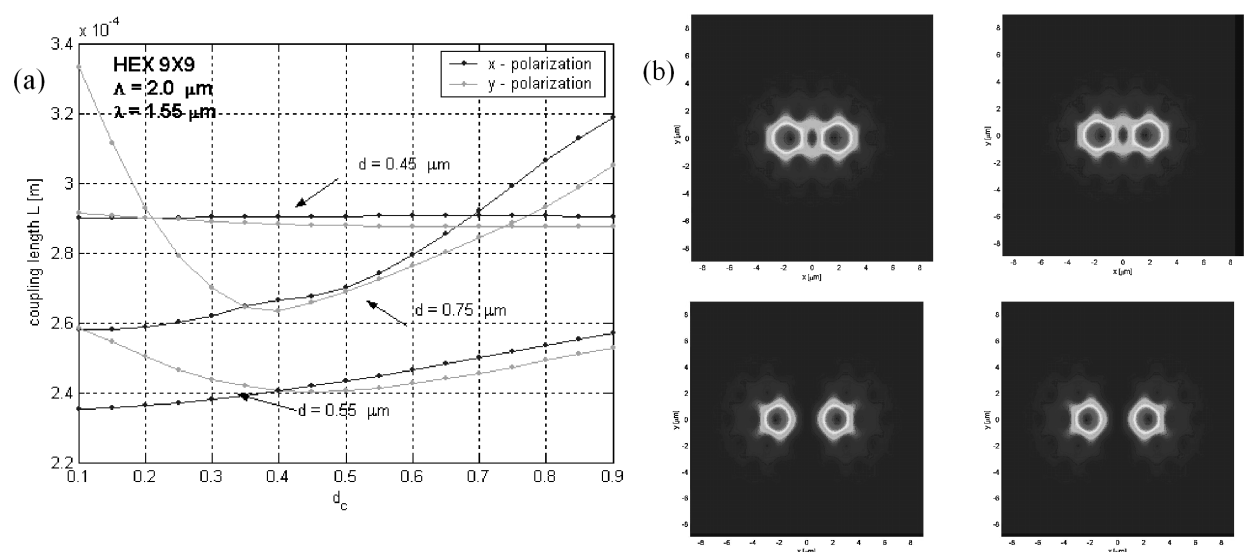

Fig. 15. (a) Coupling lengths as functions of a diameter of the central air hole for hexagonal lattice and lattice constant $\Lambda=2.0 \mu \mathrm{m}$, (b) the intensity distributions for the fundamental mode components guided in the polarization independent double core PCF: the lattice constant of $\Lambda=2.0$, filling factor $d / \Lambda=0.3$ and the central hole diameter is $d_{\mathrm{c}}=0.4 \mu \mathrm{m}, \lambda=1.55 \mu \mathrm{m}, L_{x y}=241 \mu \mathrm{m}$. 


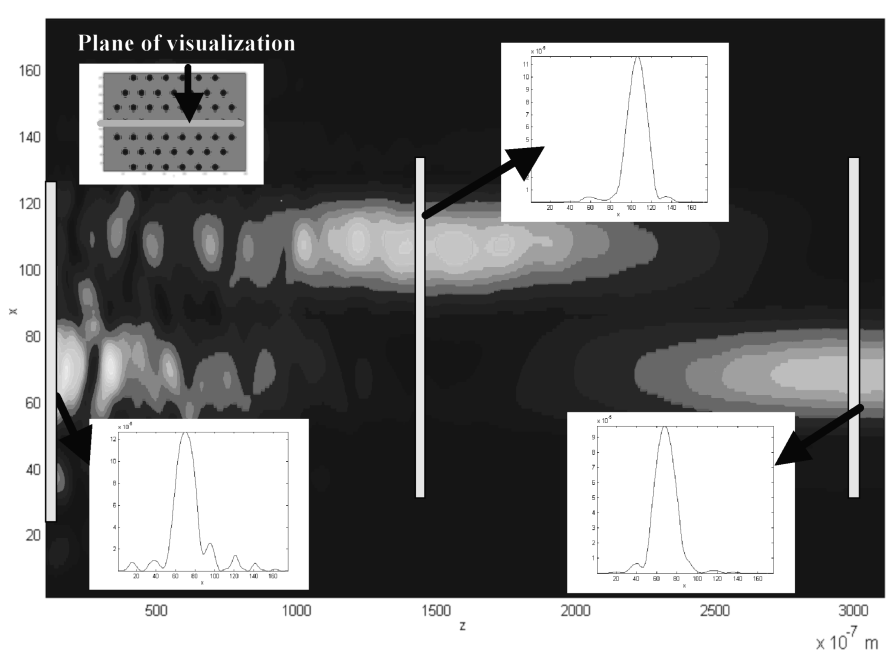

Fig. 16. FDTD simulation of directional coupling in the polarization-independent double-core PCF. The input beam is polarized at 45 degrees with respect to the main axis to stimulate both orthogonal polarizations.

the same coupling lengths for both polarizations. This way polarization independent double-core coupling is obtained (Figs. 15 and 16).

\subsection{Highly birefringent fibers}

In the bow tie and panda type optical fibers, high birefringence comes from the mechanical stresses in the core, while in fibers with an elliptical cross-section it is a result of the broken axial symmetry of the structure. The birefringence of the photonic crystal fibers is obtained due to non-axisymmetric distribution of the effective refraction index that depends on the size and spatial distribution of holes [41-44]. The interest in highly birefringent PCF is growing because of its expected extremely high birefringence in comparison to standard optical fibers.

In PCF with a hexagonal lattice and circular air-holes, the symmetry has the order of $m=6$. Steel et al. [45] show that in such PCFs the fundamental mode is degenerated and the fibers are not birefringent. Steel \& Osgood [46] predicted high birefringence in fibers with elliptical holes, where the symmetry of the structure is $m=2$. Szpulak et al. show another structure of PCF where one of the polarization components of the fundamental mode is highly attenuated and it can be used as a polarization fiber over an extremely wide range of spectrum [47].

The symmetry of the order $m=2$ in fibers can be obtained in different structures: hexagonal lattice with elliptical holes, rectangular lattice with circular holes, hexagonal lattice with circular holes, and elliptical core region (Fig. 17). The degeneracy of the fundamental mode is then lifted and the two polarized modes $H E_{11}^{x}$ and $H E_{11}^{y}$ are carried with different propagation constants. 

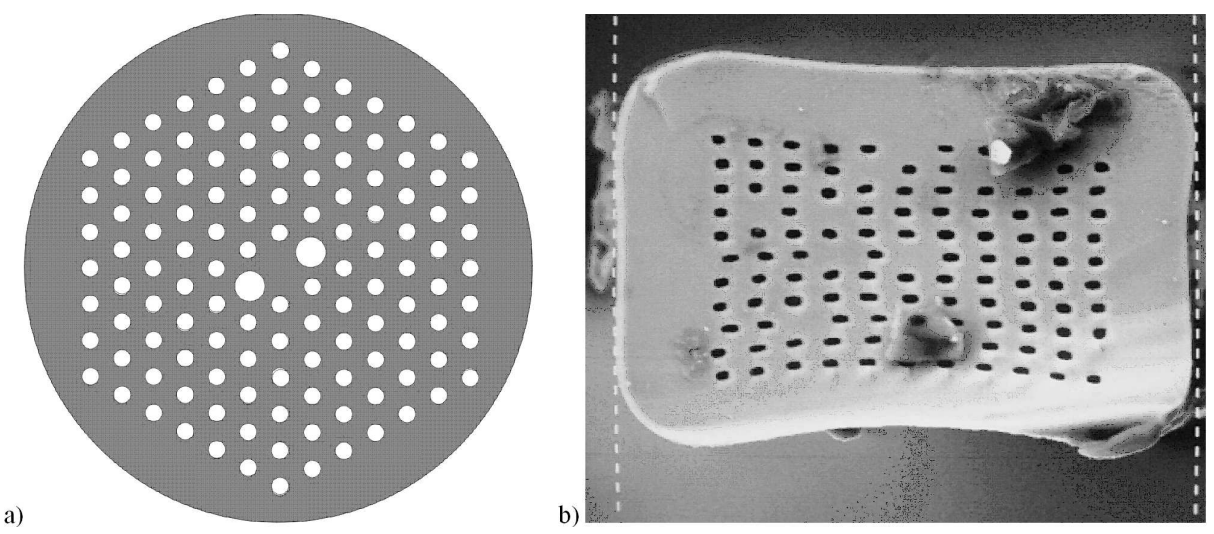

Fig. 17. Examples of highly birefringent PCF: (a) HB PCF with hexagonal lattice and circular holes (after Suzuki et al. [44]), (b) test samples of rectangular-shape HB PCF with rectangular lattice and elliptical holes of IEMT.

The birefringence $B$ is defined as a difference between the propagation constants $\beta_{x}$ and $\beta_{y}$ of the two orthogonal polarized components $H E_{11}^{x}$ and $H E_{11}^{y}$ of the fundamental mode:

$$
B=\lambda\left(\beta_{x}-\beta_{y}\right) / 2 \pi=\left|n_{\mathrm{eff} x}-n_{\mathrm{eff} y}\right| .
$$

Many works have shown that in structures with a hexagonal and rectangular lattice, the birefringence increases with decreasing normalized frequency $\Lambda_{x} / \lambda$. For $\Lambda_{x} / \lambda=0.8$, the birefringence in a structure with a rectangular lattice may reach theoretically values of the order $10^{-2}$. The modeled rectangular structure with the parameters $\Lambda_{x}=1.24 \mu \mathrm{m}, \Lambda_{y}=0.96 \mu \mathrm{m}, l_{x}=1 \mu \mathrm{m}, l_{y}=0.51 \mu \mathrm{m}$ shows

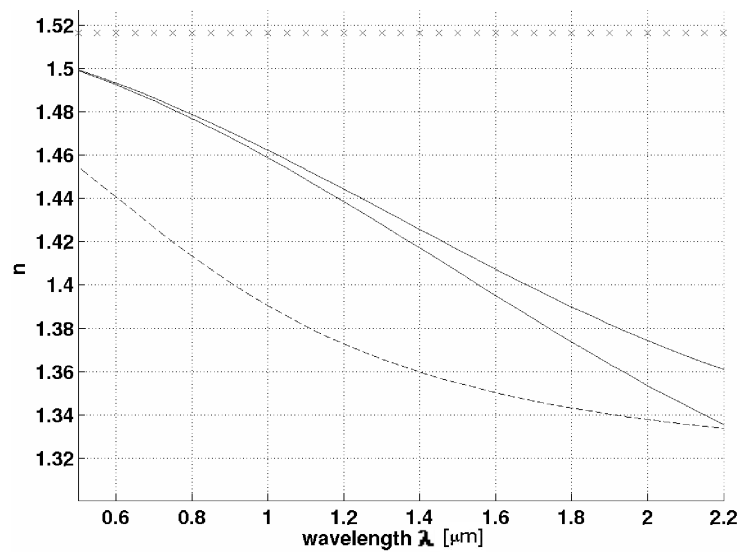

Fig. 18. The effective refraction index for modes carried by a photonic crystal fiber with lattice constants $\Lambda_{x}=1.24 \mu \mathrm{m}, \Lambda_{y}=0.96 \mu \mathrm{m}$ and holes $l_{x}=1 \mu \mathrm{m}, l_{y}=0.51 \mu \mathrm{m}$. Solid lines correspond to carried modes, dashed and dotted lines represent the photonic cladding effective index and the refraction index. 


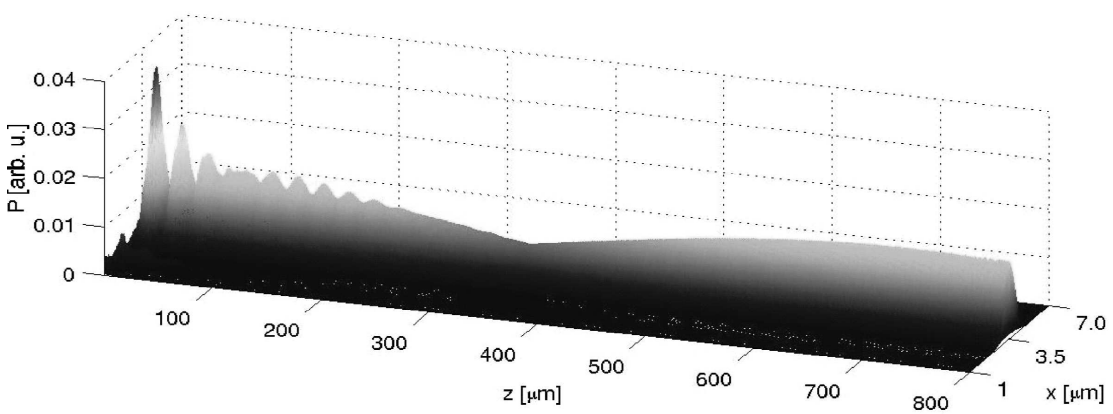

Fig. 19. Temporal length of Poyting vector in the steady state. Input light: linearly polarized at $45^{\circ}$ with respect to main axis of the fiber - 3D FDTD simulation of highly birefringent PCF.

the birefringence $B=1.1 \times 10^{-2}$ and carries only the fundamental mode. The effective refractive index of polarization components and the distribution of the field over the fiber's cross-section are shown in Figs. 18 and 19.

Since the birefringence in PCFs comes from non-axisymmetric distribution of the effective refraction around the core, they are highly insensitive to temperature. Due to this immunity highly birefringent PCFs are very attractive for sensing and for telecommunication applications as a compensator of polarization mode dispersion in fiber lines.

\subsection{Nonlinear fibers and supercontinuum generation}

A PCF with a very small core can exhibit very high nonlinear properties. The possibility of a combination of very small core sizes (diameters down to $1 \mu \mathrm{m}$ ) and a very large core/cladding index contrast (up to 0.4 ) enables us to create fibers with extremely small effective areas and high nonlinear coefficients (Fig. 20). The low index of the cladding is obtained by an extremely high filling fraction, where more than $90 \%$ of the cladding is filled with air. In such fiber light is strongly confined in the small core. Moreover, the dispersion characteristics can be designed to make different nonlinear effects possible. It makes a nonlinear PCF a promising medium for four-wave mixing, Raman amplification, optical parametric amplification and supercontinuum generation for metrology, optical coherence tomography (OCT) or spectroscopy. To obtain soliton transmission, dispersion and nonlinearity have to be balanced. PCFs allow one to modify its dispersion properties in an extremely large range, including anomalous dispersion below one micron wavelength and size of the core, which alters effectively nonlinearity of the fiber. Wadsworth et al. reported on soliton propagation at $850 \mathrm{~nm}$ over up to $20 \mathrm{~m}$ of fiber. The fiber has

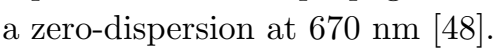

PCF fibers have already proven to be a very efficient medium for supercontinuum generation. Supercontinuum generation was first observed in 1970 and has since then been studied extensively in many different materials. 

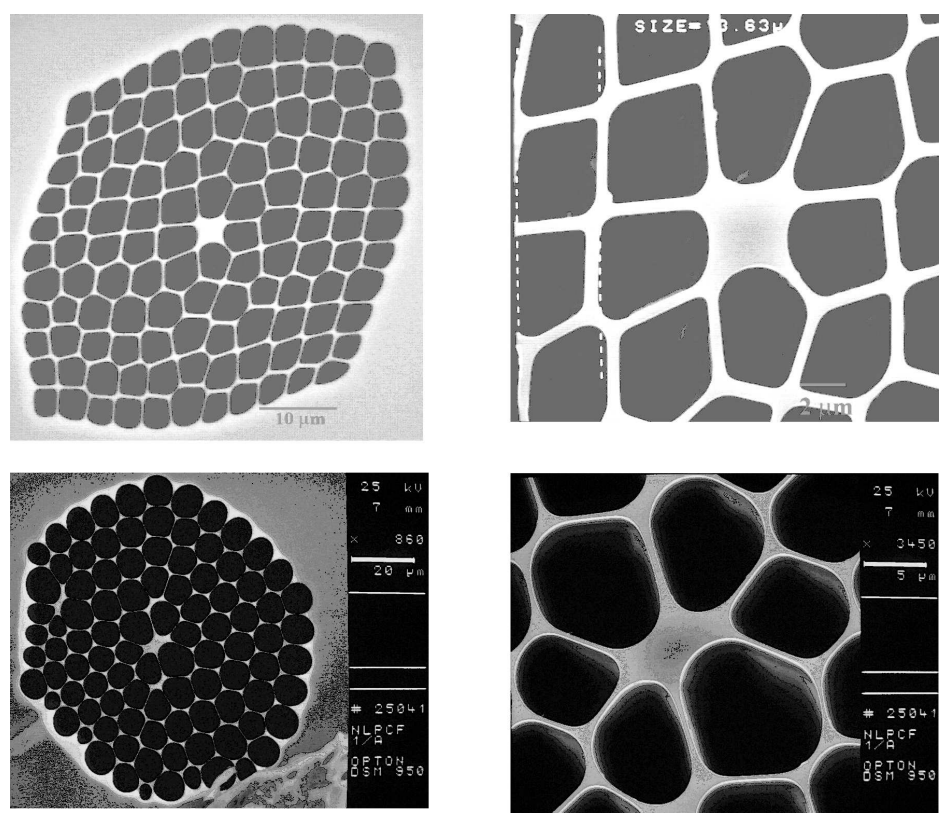

Fig. 20. Nonlinear PCFs have very small core sizes and a very large core/cladding index contrast due to a large air fraction. Samples of nonlinear PCF fabricated at the Institute of Electronic Materials Technology in Warsaw. The fibers have cores of diameter 1-3 $\mu \mathrm{m}$, air holes diameters are $3-5 \mu \mathrm{m}$ and the fiber diameters are $200-270 \mu \mathrm{m}$.

Supercontinuum generation is a result of several different nonlinear phenomena like self-phase modulation, Raman scattering, phase matching, and solitons. In principle it is generation of pulse with significant spectral broadening with respect to optical pump pulses. The supercontinuum effect has been reported in PCFs with pumping in the anomalous dispersion regime or at the zero-dispersion wavelength in both the visible and the infrared wavelength range.

Most experiments utilize femtosecond pumping as this results in extremely broad spectra, from 400 to beyond $1750 \mathrm{~nm}$ [49]. Picosecond pulse pumping gives more narrow spectra but since the laser source is cheaper in this case it attracts practical implementation. Andersen et al. obtained from 2.5 ps pump impulse a wavelength spectra from 1500 to $1620 \mathrm{~nm}$ [50]. Generation of supercontinuum in this region can find application in wave division multiplexing systems as the realization of multi-wavelength short pulse sources or wavelength converters for multicasting. The nonlinear coefficient is reported in the range of $30-70 \mathrm{~W}^{-1} \cdot \mathrm{km}^{-1}$ at $1.55 \mu \mathrm{m}$.

PCFs are very promising media for supercontinuum generation since dispersion characteristics can be designed to obtain the generation in a specific region. It allows us to convert light to both higher and lower wavelengths in the octave range with relatively low power levels. 


\subsection{Fiber lasers and amplifiers}

Fiber amplifiers are one of the key components of modern telecommunication. Also fiber lasers are starting to be more and more important in medicine, spectroscopy, and industry. Compared to conventional solid-state lasers, the great advantage of fiber lasers is their outstanding heat-dissipation capability. It results from the large ratio of fiber surface to volume in a long, thin gain medium. Beam parameters depend only on design of the fiber and its quality is not perturbed with thermal distortion. Such fibers can be pumped by multimode laser diodes and provide lasing/amplification action in a single mode.

Conventional SIFs for lasers consist of core and double cladding made of different materials - most typically with a polymer outer cladding. Efficiency of these devices is limited by core size, numerical aperture, and Raman scattering in doped silica. It results in limited output power that such a device can deliver.

Double clad of PCF is made of silica with two photonic claddings with different properties. The inner cladding ensures a high numerical aperture and is surrounded with a web of silica bridges which are substantially narrower than the wavelength of the guided radiation. The rare-earth ion doping medium of a fiber laser such as $\mathrm{Yb}, \mathrm{Nd}, \mathrm{Er}, \mathrm{Er} / \mathrm{Yb}, \mathrm{Tm}$, Ho is introduced into the core of the PCF (Fig. 21). Numerical apertures of 0.8 of double clad PCFs are reported [51]. The benefit of such an air-clad fiber with a high NA is that the diameter of the inner cladding (pump core) can be significantly reduced while brightness acceptance of the pump radiation is kept. Due to the increased ratio of active core area to inner cladding (pump core), the pump light absorption is improved. It allows us to use inexpensive, high power broad area emitting pumps. Large mode area for the

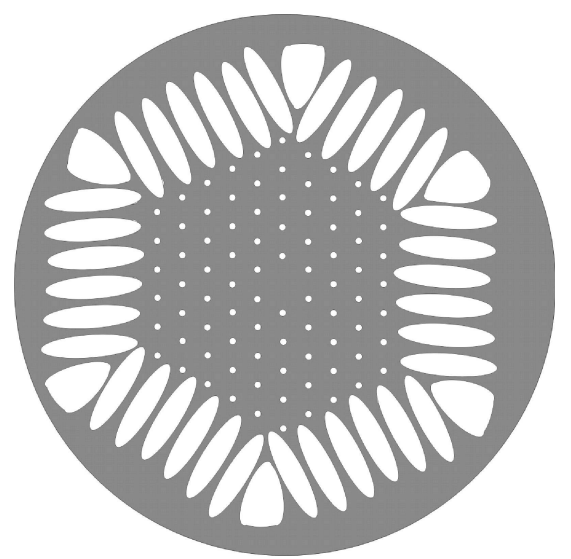

Fig. 21. Double clad PCF (after Limpert et al. [51]). A solid core is surrounded with low filling factor cladding (inner one), which plays a role of a pump core since the pump field is confined by a second high filling factor cladding (outer one). 
single mode signal allows one to obtain a high power output with relatively low power density. This way nonlinear phenomena and fiber damage due to overheating can be avoided. However problems with heat dissipation of the fiber may occur limiting high power performance, since air-holes thermally isolate the core from the environment. On the other hand no radiation has a direct contact with the coating material. This makes these fibers predestinated for high power operations.

Doping with photosensitive materials makes also possible the fiber Bragg grating inscription for all-fiber laser development. Limpert et al. [51] reported laser generating up to $80 \mathrm{~W}$ output power with a $2.3 \mathrm{~m}$ long air-clad ytterbiumdoped large-mode area photonic crystal fiber. The slope efficiency is $78 \%$ and single transverse mode operation is achieved with a mode-field area of $350 \mu \mathrm{m}^{2}$. The core is triangularly shaped with a diameter of about $28 \mu \mathrm{m}$ with a numerical aperture of 0.05 , while the doped rods have a diameter of $9 \mu \mathrm{m}$. The diameter of the inner cladding is $150 \mu \mathrm{m}$ with a numerical aperture of 0.55 . The extracted power per fiber length $(\sim 35 \mathrm{~W} / \mathrm{m})$ is higher than that reported for conventional double-clad high power fiber lasers. Air-clad microstructure ytterbium-doped large-mode-area fibers are predicted to be scalable to $\mathrm{kW}$-level output powers, where nonlinearity will limit the performance [52].

\subsection{Imaging with PCF fibers}

A specially designed PCF can be used as a flexible imaging fiber as a replacement of fiber imaging bundles. Imaging fiber bundles are used in imaging inaccessible areas, such as inside the human body, nuclear reactors, and jet engines and to interconnect $2 \mathrm{D}$ arrays in back-plane communication systems. It takes a lot of effort to fabricate bundles with regular size and position of individual cores and high capturing fraction. Additionally, a minimum diameter of the bundle is at least a few millimeters, which limits their use in some applications (e.g. in endoscopy of small body cavities). The PCF technique was successfully used to fabricate a new, sub-millimeter diameter, imaging flexible fiber. Two different guiding mechanisms are explored, solid core and air core guidance. Both mechanisms make the transmission of coherent image possible and are demonstrated by Eijkelenborg [53]. A microstructured fiber with $800 \mu \mathrm{m}$ diameter and 112 air cores with a spacing $42 \mu \mathrm{m}$ was fabricated and tested experimentally.

\subsection{Fresnel fiber}

A lot of interest is paid to overcome the free-space diffraction limit in propagation of high intensity light. An example of diffractionless free-space waveform is a Bessel wave. It can be described as a series of Gaussian waves that propagate along a forward axis such that the superposition forms the peak intensity in the middle and the distribution approaching that of a Bessel wave. In practice, it is very difficult to generate these waves beyond the Raighley range of conventional optics. The microstructured fiber technique allows one to fabricate a fiber with 


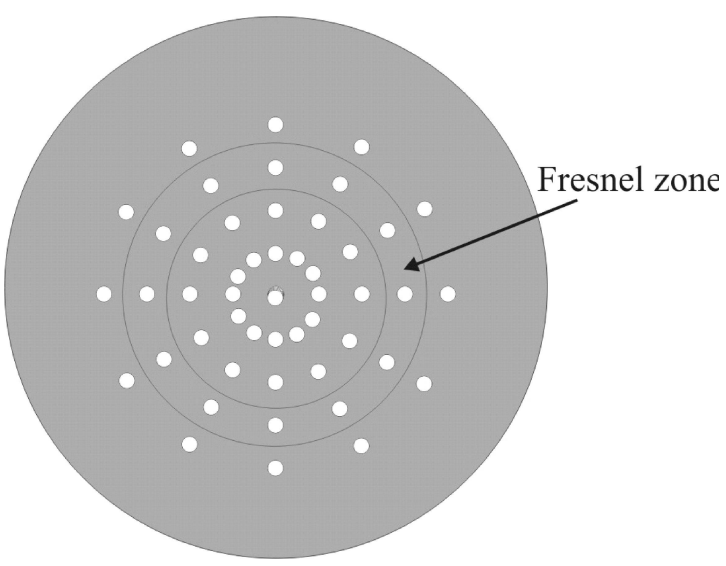

Fig. 22. A concept of the Fresnel fiber after Canning et al. [54].

Fresnel zones determined by the ring of holes spaced at radii such that interstitial hole spacing can be significantly larger than the propagation wavelength. The concentric rings of holes (Fresnel zones) have various effective refractive indexes [54] (Fig. 22). This way light passing through the fiber scatters on surfaces of air holes and interferes constructively, forming a peak field intensity in the center of the fiber axis. This enables focusing light at the output of the fiber at the far field without any additional lens, while in conventional fibers, light emerging from a fiber diffracts and expands.

In general, the generation of optical wave guide propagation similar to the free space propagation enables possible solutions to controlling and shaping optical field generation in the free space using coherent scattering of multiple sources [55].

\section{Conclusions}

Photonic crystal fibers combine properties of $2 \mathrm{D}$ photonic crystals and classical fibers. Research on photonic crystal fibers is still very young and we may expect many new developments, more accurate and efficient methods for modeling and characterization. Mechanisms of guidance based on photonic bandgap have been implemented and tested. Several new interesting properties such as hollow-core guidance and endlessly single mode fibers, tuning properties with liquid crystals have been investigated. Dispersion engineering is possible in PCFs in the range unachievable for classical fibers (flat dispersion in the large range, zero-dispersion, and anomalous dispersion in the visible range).

It is still difficult to estimate the impact of PCF on development of photonics. Apart from already developed applications as fiber dispersion compensation, supercontinuum generation, and particle guidance we can expect a series of new applications in telecommunication, sensing, beam delivery, surgery, spectroscopy, and fiber lasers in the next few years. 


\section{Acknowledgments}

This work was supported by the research grant 4T11B05123 of the State Committee for Scientific Research. Some of the modeling results were obtained using computer resources of the Interdisciplinary Centre for Mathematical and Computational Modelling (ICM), Warsaw University.

\section{References}

[1] P. Yeh, A. Yariv, E. Marom, J. Opt. Soc. Am. 68, 1196 (1978).

[2] J.C. Knight, T.A. Birks, P.St.J. Russell, D.M. Atkin, Opt. Lett. 21, 1547 (1996).

[3] J.C. Knight, J. Broeng, T.A. Birks, P.S. Russel, Science 282, 1476 (1998).

[4] P.St. Russel, Science 299, 358 (2003).

[5] R.F. Cregan, B.J. Mangan, J.C. Knight, T.A. Birks, P.S. Russell, P.J. Roberts, D.C. Allan, Science 285, 1537 (1999).

[6] K. Kiang, K. Frampton, T. Monro, R. Moore, J. Tucknott, D. Hevak, N. Broderick, D. Richardson, H. Rutt, Electron. Lett. 38, 546 (2002).

[7] R. Stepien, L. Kociszewski, D. Pysz, Proc. SPIE 3570, 62 (1998).

[8] D. Pysz, R. Stepien, P. Szarniak, R. Buczynski, T. Szoplik, Proc. SPIE 5576, 78 (2004)

[9] T. Monro, Y. West, D. Hevak, N. Broderick, D. Richardson, Electron. Lett. 36, 1998 (2000).

[10] V. Ravi Kanth Kumar, A. George, J. Knighr, P. Russell, Opt. Expr. 11, 2641 (2003).

[11] A. Taflove, S. Hagness, Computational Electrodynamics: The Finite Difference Time-Domain Method, Artech House, Boston 2000.

[12] F. Fogli, G. Bellanca, P. Bassi, I. Madden, W. Johnstone, IEEE J. Lightwave Technol. 17, 136 (1999).

[13] Z. Zhu, T.G. Brown, Opt. Expr. 10, 853 (2002).

[14] E. Silvestre, M.V. Andres, P. Andres, IEEE J. Lightwave Technol. 16, 923 (1998).

[15] A. Ferrando, E. Silvestre, J.J. Miret, P. Andres, M.V. Andres, Opt. Lett. 24, 276 (1999).

[16] D. Mogilevtsev, T.A. Birks, P.St.J. Russell, J. Lightwave Technol. 17, 2078 (2001).

[17] W. Zhi, R. Guobin, L. Shuqin, J. Shuisheng, Opt. Expr. 11, 980 (2003).

[18] A. Cucinotta, S. Selleri, L. Vincetti, M. Zoboli, IEEE Photon. Technol. Lett. 14, 1530 (2002).

[19] P. White, R.C. McPhedran, L.C. Botten, G.H. Smith, C.M. de Sterke, Opt. Expr. 9, 721 (2001).

[20] R. Scarmozzino, A. Gopinath, R. Pregla, S. Helfert, IEEE J. Selected Topics in Quantum Electronics 6, 150 (2000).

[21] T.A. Birks, J.C. Knight, P.St.J. Russell, Opt. Lett. 22, 961 (1997).

[22] J.C. Knight, T.A. Birks, P.St.J. Russel, J.P. de Sandro, JOSA A 15, 748 (1998). 
[23] J. Bagget, T. Monro, K. Furusawa, D. Richardson, Opt. Lett. 26, 1045 (2001).

[24] A. Ferrando, E. Silvestre, P. Andres, J.J. Miret, M.V. Andres, Opt. Expr. 9, 678 (2001).

[25] H. Reeves, J. Knight, P. Russell, P. Roberts, Opt. Expr. 10, 609 (2002).

[26] K. Hansen, Opt. Expr. 11, 1503 (2003).

[27] K. Saitoh, M. Koshiba, T. Hasegawa, E. Sasaoka, Opt. Expr. 11, 843 (2003).

[28] J. Broeng, T. Sondergaard, S. Barkou, P. Barbeito, A. Bjarklev, J. Opt. A; Pure Appl. Opt. 1, 477 (1999).

[29] G. Vienne, Y. Xu, C. Jakobsen, H.J. Deyerl, T.P. Hansen, B.H. Larsen, J.B. Jensen, T. Sorensen, M. Terrel, Y. Huang, R. Lee, N.A. Mortensen, J. Broeng, H. Simonsen, A. Bjarklev, A. Yariv, in: Proc. Optical Fiber Communication Conf. (OFC) 2004, OSA Proc. Series, Vol. 95, Optical Society of America, Washington (D.C.) 2004, Paper PDP25.

[30] B.J. Mangan, L. Farr, A. Langford, P.J. Roberts, D.P. Williams, F. Couny, M. Lawman, M. Mason, S. Coupland, R. Flea, H. Sabert, T.A. Birks, J.C. Knight, P.St.J. Russell, in: Proc. Optical Fiber Communication Conf. (OFC) 2004, OSA Proc. Series, Vol. 95, Optical Society of America, Washington (D.C.) 2004, Paper PDP24.

[31] N. Venkataraman, M.T. Gallagher, C.M. Smith, D. Müller, J.A. West, K.W. Koch, J.C. Fajardo, in: Proc. ECOC 2002, Copenhagen (Denmark) 2002, Paper PD1.1.

[32] K. Nakayama, T. Saitoh, M. Kahui, K. Kawasaki, M. Matsui, H. Takamizawa, H. Miyaki, in: Proc. Optical Fiber Communication Conf. (OFC) 2002, OSA Proc. Series, Vol. 70, Optical Society of America, Washington (D.C.) 2002, postdeadline paper FA10.

[33] D.G. Ouzounov, F.R. Ahmad, D. Müller, N. Venkataraman, M.T. Gallagher, M.G. Thomas, J. Silcox, K.W. Koch, A.L. Gaeta, Science 301, 1702 (2003).

[34] T. Larsen, D. Hermann, J. Broeng, A. Bjarklev, in: 29th European Conf. on Optical Communication, ECOC'03, Rimini (Italy) 2003, Associazione Elettrotecnica et Elettronica Italiana, p. 39.

[35] F. Benabid, J.C. Knight, P. Russell, Opt. Expr. 10, 1195 (2002).

[36] B.J. Mangan, J.C. Knight, T.A. Birks, P.S. Russell, A.H. Greenaway, Electron. Lett. 36, 1358 (2000).

[37] G. Kakarantzas, A. Ortigosa-Blanch, T.A. Birks, P.S. Russell, L. Farr, F. Couny, B.J. Mangan, Opt. Lett. 28, 158 (2003).

[38] P.M. Blanchard, J.G. Bernett, G.R.G. Erry, A.H. Greenaway, P. Harrison, B. Mangan, J.C. Knight, P.S. Russell, M.J. Gander, R. McBride, J.D.C. Jones, Smart Matter Struc. 9, 132 (2000).

[39] W. MacPherson, M.J. Gander, R. McBride, J.D.C. Jones, P.M. Blanchard, J.G. Burnett, A.H. Greenaway, B. Mangan, T.A. Birks, J.C. Knight, P.St.J. Russell, Opt. Commun. 193, 97 (2001).

[40] L. Zhang, C. Yang, Opt. Expr. 11, 1015 (2003).

[41] A. Ortigosa-Blanch, J.C. Knight, W.J. Wadsworth, J. Arriaga, B.J. Mangan, T.A. Birks, P.St.J. Russell, Opt. Lett. 25, 1325 (2000). 
[42] T.P. Hansen, J. Broeng, S.E.B. Libori, E. Knudsen, A. Bjarklev, J.R. Jensen, H. Simonsen, IEEE Phot. Techn. Lett. 13, 588 (2001).

[43] K. Saitoh, M. Koshiba, IEEE Phot. Techn. Lett. 14, 1291 (2002).

[44] K. Suzuki, H. Kubota, S. Kawanishi, Opt. Expr. 9, 676 (2001).

[45] M.J. Steel, T.P. White, C. Martijn de Sterke, R.C. McPhedran, L.C. Botten, Opt. Lett. 26, 488 (2001).

[46] M.J. Steel, R.M. Osgood, Jr., Opt. Lett. 26, 229 (2001).

[47] M. Szpulak, T. Martynkien, W. Urbanczyk, in: Proc. 4th Intern. Conf. on Transparent Optical Networks: ICTON 2004, Wroclaw (Poland) 2004, National Institute of Telecomunication, Warsaw 2004, p. 91.

[48] W. Wadsworth, J. Knight, A. Ortigosa-Blanch, J. Arriaga, E. Silvestre, P. Russell, Electron. Lett. 26, 53 (2000).

[49] H. Hundertmark, D. Kracht, D. Wandt, C. Fallnich, V.V. Ravi Kanth Kumar, A.K. George, J.C. Knight, P.S.J. Russell, Opt. Expr. 11, 3196 (2003).

[50] A. Andersen, C. Peucheret, K. Hilligsoe, K. Berg, K. Hansen, P. Jeppesen, in: Proc. 5th Intern. Conf. on Transparent Optical Networks: ICTON 2003, Warsaw (Poland) 2003, National Institute of Telecomunications, Warsaw 2003, p. 66.

[51] J. Limpert, T. Schreiber, S. Nolte, H. Zellmer, A. Tunnermann, R. Iliew, F. Lederer, J. Broeng, G. Vienne, A. Petersson, C. Jakobsen, Opt. Expr. 11, 818 (2003).

[52] J. Limpert, A. Liem, T. Schreiber, F. Röser, H. Zellmer, A. Tünnermann, Photonic Spectra, May 2004.

[53] M. Eijkelenborg, Opt. Expr. 12, 342 (2004).

[54] J. Canning, E. Buckley, K. Lyytikainen, Opt. Lett. 28, 230 (2003).

[55] J. Canning, E. Buckley, K. Lyytikainen, Opt. Expr. 11, 347 (2003). 